\title{
In vitro and Short-term in vivo Characteristics of a Kel-F Thin Film Modified Glucose Sensor
}

\author{
Sun Kil Kang, ${ }^{*}$ Ran-A Jeong, ${ }^{*}$ Sejin Park, $*$ Taek Dong Chung, ${ }^{* *}$ Sunmin Park,*** \\ and Hee Chan KIM*i \\ *Department of Biomedical Engineering, College of Medicine and Institute of Medical and Biological \\ Engineering, Medical Research Center, Seoul National University, Seoul 110-744, Korea \\ **Department of Chemistry, Sungshin Women's University, Seoul 136-742, Korea \\ ***Department of Food and Nutrition, Hoseo University, Asan 336-795, Korea
}

\begin{abstract}
A new outer layer composition, consisting of polytetrafluoroethylene (PTFE), Kel-F oil, and Nafion, is suggested to minimize the detrimental effect of dissolved oxygen and to extend the linear response range of a glucose oxidase(GOx)based sensor using nonconducting polymer. The morphology of Kel-F/PTFE/Kel-F/Nafion polymeric laminate was followed during fabrication by SEM. When Kel-F film was formed on the PTFE outer layer, the linear response was extended to $21 \mathrm{mM}$, at a sensitivity of $2.8 \pm 0.8 \mathrm{nA} / \mathrm{mM} \mathrm{mm}^{2}$. We demonstrate that a sensor without Kel-F/PTFE/KelF/Nafion outer layer is relatively oxygen dependent, whereas by comparison a sensor with Kel-F/PTFE/Kel-F/Nafion outer layer is oxygen independent. The current of such a glucose sensor implanted in the subcutaneous tissue stabilized within $60 \mathrm{~min}$, and the lag between blood glucose changes and sensor output was within $1 \mathrm{~min}$. The in vivo characteristics of the glucose sensor described show great promise for one-point in vivo calibration.
\end{abstract}

(Received June 2, 2003; Accepted July 25, 2003)

\section{Introduction}

The enzyme-based biosensors for continuous glucose monitoring have long been investigated as a promising method of improving glycemic control in persons with diabetes. However, in vitro and in vivo functions of sensors still remain to be further improved to allow wide-spread use of an implantable real-time sensor.

First of all, as for a GOx-based sensor, which requires oxygen as a co-substrate to carry out glucose oxidation, it is important to examine the so-called "oxygen effect", which means the dependence of the sensor performance on the oxygen supply. Zhang et al. ${ }^{1}$ demonstrated that the demand for oxygen by the sensor is primarily determined by its sensitivity, because the absolute current output is a direct measure of the rate of oxygen consumption. Non-linear sensors exhibit a strong oxygen dependence because the overall process depends on oxygen and glucose fluxes. Many efforts to minimize the oxygen effect have included the use of proper membrane coverage that improves the surface availability of oxygen relative to glucose. Gough et al. ${ }^{2}$ reported a novel sensor configuration in which oxygen diffuses into the membrane from two directions while glucose diffuses from only one. Wang and $\mathrm{Lu}^{3}$ used Kel-F oil, which has very high oxygen solubility, as an internal supply of oxygen for a carbon paste electrode. However, this type of sensor has not yet been successfully implanted in a body to monitor glucose concentration in diabetes. Zhang et al. constructed a practically useful implantable glucose sensor, which was essentially free of oxygen fluctuation, by controlling

$\doteqdot$ To whom correspondence should be addressed.

E-mail: hckim@snu.ac.kr the sensor's sensitivity and linearity. Sensitivity was controlled by the thickness of an outer polyurethane membrane. The outer layer served to increase the relative oxygen supply because it functioned only as a diffusion barrier to glucose, while it imposed little or no effect on oxygen diffusion.

The second issue is to develop a reproducible and predictable method for sensor fabrication. When an enzyme layer is prepared on a miniaturized/microfabricated needle or a microfabricated electrode, electropolymerization has valuable advantages over solvent casting because it is not limited in terms of the geometry and area of the electrode. The method also offers a site-selectivity that makes it suitable for the development of a multianalyte-sensing system, and the uniformity of the polymer film on the electrode surface can cope with more complex geometries. ${ }^{4,5}$ Yang et al. fabricated a miniaturized glucose sensor using microfabrication and electropolymerization techniques. ${ }^{6}$ Glucose oxidase (GOx) was entrapped during the electropolymerization of a 1,3diaminobenzene (1,3-DAB) on a recessed rectangular microfabricated platinum electrode. On the other hand, many GOx-based sensors prepared by electropolymerization have suffered from problems due to a restricted linear response range, though they have shown high sensitivities and fast responses. As was demonstrated in the study reported by Zhang et al., the restricted linear response range results from a stoichiometric limitation of the enzyme reaction by oxygen. Therefore, the glucose sensitivity of a sensor should be adjusted by applying an outer membrane to extend the linear response range of a sensor. ${ }^{7}$ Yang et al. extended the range of linear response to higher concentration by using Teflon and polyurethane film on a recessed rectangular microfabricated platinum electrode as an outer layer.

As the third issue of biocompatibility, in spite of the very high 
number of papers concerning enzyme immobilization in electrogenerated polymeric films, relatively few have successfully addressed the fabrication and characterization of implantable needle-type glucose sensors for practical in vivo applications. ${ }^{8}$ In a previous study, ${ }^{6,9}$ we used PTFE as a permselective diffusion-limiting biocompatible outer layer on the enzyme layer, consisting of poly(1,3-DAB), glutaraldehyde, poly-L-lysine, and GOx. It was demonstrated that PTFE dispersed in aqueous media could be applied as an outer membrane on the electropolymerized enzyme layer. In spite of the improved linear response range of the sensor, however, it had unsatisfactory linearity, mechanical stability, and biocompatibility, which prevented it from being implanted in vivo.

We report here upon studies on the construction of a new outer layer, based on a Kel-F thin film. The film was used to extend the linear response range as well as to minimize the oxygen effect of the glucose sensor. To enhance the biocompatibility of the sensor, Nafion, which has been widely used as a biocompatible layer, was coated as an outer film in this study. Finally, the in vivo performance was evaluated using the finished sensor. Morphological changes of the electrode surface during sensor fabrication and the cross sections of Pt/poly(1,3-DAB), glutaraldehyde, poly-L-lysine, and GOx/KelF/PTFE/Kel-F/Nafion were determined by SEM.

\section{Experimental}

\section{Materials}

Glucose oxidase (E.C. 1.1.3.4. high purity, 250000 unit/g, without $\mathrm{O}_{2}$ type II-S, Argellius niger), D-(+)-glucose, and acetaminophen (AP) were used as supplied by Sigma. LAscorbic acid, 1,3-diaminobenzene (1,3-DAB, 99+\%), glutaraldehyde $(25 \mathrm{wt} \%$ solution in water), dispersed polytetrafluoroethylene in water $(60 \%$, PTFE solution), and Nafion were purchased from Aldrich and used without further purification. Poly-L-lysine (hydrobromide salt with molecular weight, 23400) was used after it had been dialyzed in $0.1 \mathrm{M}$ $\mathrm{NaCl}$. Poly-L-lysine solution was stored at $4^{\circ} \mathrm{C}$ when not in use. Kel-F oil was generously donated by Powderchek (Fremont, CA), for which we are very grateful.

\section{Instrumentation}

Electrochemical experiments were performed with a Model cDAQ-1604 multichannel potentiostat (ELBIO Co., Seoul). $\mathrm{Ag} / \mathrm{AgCl}(3 \mathrm{M} \mathrm{KCl})$ and platinum wire were used as the reference and counter electrodes, respectively. Acquired data were transferred to a personal computer using USB protocol. The control and data acquisition program was written in LabVIEW software (National Instrument, version 5.0). When the outer membrane was formed, it was dehydrated in a vacuum oven (Lab-line, 3606-1CE). The surface morphologies of fabricated electrodes were examined under the scanning electron microscope (SEM) (Model JSM 840-A).

\section{Fabrication of sensors}

The glucose sensor was fabricated as reported previously by Chung et al. ${ }^{9}$ Teflon-coated platinum-iridium (Pt/Ir) alloy wires were employed as an electrode body for the needle-type of sensor. The Teflon skin of the wire was removed from the end so that the area of the exposed bare Pt/Ir surface was $1.72 \mathrm{~mm}^{2}$ (3 mm in length). The electropolymerization of the 1,3-DAB was carried out using five consecutive cycles between +0.2 and $+1.0 \mathrm{~V}$ at $2 \mathrm{mV} / \mathrm{s}$. After rinsing with deionized water, the enzyme electrode was dipped in an ethanol solution containing $20 \%$ Kel-F oil and then in a PTFE dispersion diluted to $30 \%$. The electrode was again dipped into an ethanol solution containing $20 \%$ Kel-F oil. Finally, the Nafion film was coated using a $1.5 \%$ Nafion solution. The electrode was dried under vacuum at $30^{\circ} \mathrm{C}$ for $1 \mathrm{~h}$ after each coating process.

\section{In vitro and in vivo evaluations of the sensor}

Experiments were performed in $0.1 \mathrm{M}$ phosphate buffered saline (PBS) solution containing $0.15 \mathrm{M} \mathrm{NaCl}$ and $0.1 \mathrm{~g} / \mathrm{L}$ $\mathrm{NaN}_{3}$, and also in a temperature-controllable cell containing 15 $\mathrm{ml}$ of PBS ( $\left.\mathrm{pH} 7.4,37^{\circ} \mathrm{C}\right)$. The effect of oxygen tension was investigated by bubbling oxygen-nitrogen mixtures at various partial pressures into the test solution. During the experiment, $\mathrm{pO}_{2}$ was maintained at a constant levels throughout the solution and the cell atmosphere. $\mathrm{pO}_{2}$ was measured using a DO meter (Pentagon 777, BioTel Co.). A serum test in undiluted serum and animal experiments were carried out to examine the biocompatibility of the glucose sensor. Ten male Sprague Dawley rats weighing 400 - $500 \mathrm{~g}$ were used in the in vivo studies. Each animal was anaesthetized with $50 \mathrm{mg}$ of ketamine given intramuscularly. After the neck was shaved, indwelling catheters were inserted into the jugular vein and carotid artery. To insert the glucose sensor subcutaneously, a $1.5 \mathrm{~cm}$ incision was made in the dorsal area, and the sensor was then inserted into the tunnel created by the blunt dissection. Care was taken to avoid any bleeding and no blood was observed in any tunnel during implantation. Immediately and the day after the sensor insertion, a continuous sensor recording was performed. During the first $60 \mathrm{~min}$, the sensor output was allowed to stabilize. Although initially the output was unstable, current outputs at $c a$. $1 \mathrm{~h}$ after insertion were stable. An intraveneous glucose tolerance test was then performed in the awake, unstressed, and fasting state. Wires in the sensor were connected to a potentiostat, the sensor was held at a standing potential of $0.4 \mathrm{~V}$. Currents were recorded digitally. A 50\% glucose solution (300 $\mathrm{mg} / \mathrm{kg}$ body weight) was injected into a jugular vein catheter in 20 to $30 \mathrm{~s}$, and blood samples were collected from the carotid artery. All blood samples were analyzed in duplicate using a Glucose Analyzer II (Beckman).

\section{Results and Discussion}

\section{In vitro test and scanning electron microscopy}

Figure 1a shows a schematic diagram of the glucose sensor. As reported in a previous study, ${ }^{9}$ the enzyme layer was deposited by cycling the potential between 0.2 and $1.0 \mathrm{~V}$ in a 1,3-DAB solution containing GOx, glutaraldehyde, and poly-Llysine, whereas the outer layer was formed by dip-coating in a PTFE, Kel-F oil, and Nafion solution. Unlike the previous layer structure, the Kel-F and Nafion films were added as a glucose diffusion limiting membrane and a biocompatible layer, respectively. Although the SEM cross sectional image (Fig. 1b) does not discriminate between the individual layers, it shows that the total thickness of the layers is $c a .3 \mu \mathrm{m}$. As indicated in Fig. 1(b), the dark layer in the lower part is PTFE of thickness ca. $2 \mu \mathrm{m}$ and the bright layer on the top of the PTFE layer is assigned to be the Kel-F plus Nafion layer.

The effect of the Kel-F coating as a glucose diffusion barrier is shown in Fig. 2. The linear response range was extended to $21 \mathrm{mM}$ whereas the sensitivity became much lower when the Kel-F film was coated onto the PTFE outer layer, which is porous and has some defects. This result corresponds to the thinking of Zhang et al., who adjusted glucose sensitivity by 


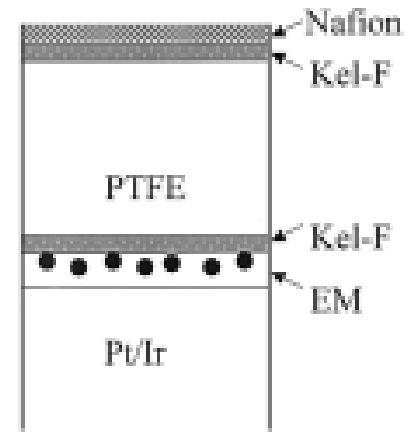

(a)

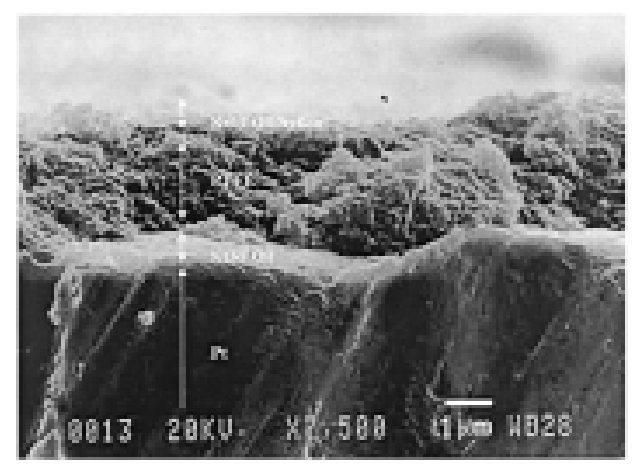

(b)

Fig. 1 (a) A schematic diagram of the constructed glucose sensor. (b) A SEM image of the cross section of Pt-Ir/poly(1,3-DAB), GOx, glutaraldehyde, poly-L-lysine/Kel-F/PTFE/Kel-F, the surface of which is shown in Fig. 3(e).

using a polyurethane membrane in a GOx-based glucose sensor. The Kel-F coating decreased the diffusion rate of glucose into the enzyme layer, but left the oxygen diffusion unaffected, resulting in an increased relative oxygen supply. Perfluorocarbon polymer layers have recently been reported to serve as a glucose diffusion limiting membrane and offer the prospect of enzyme-based biosensors with excellent longevities. ${ }^{10}$

Figure 3 shows morphology changes of the electrode surface during the sensor fabrication. Electrodeposition of 1,3-DAB gives a particulate film immobilizing GOx (Fig. 3b). The enzyme layer was so thin that surface cracks of several tens of nanometers on the bare $\mathrm{Pt}$ surface (Fig. 3a) remained visible even after the layer had been formed. In a previous report, ${ }^{9}$ the film thickness appeared to be $54 \mathrm{~nm}$ from an EQCM study. When Kel-F oil was cast on a poly(1,3-DAB) layer, it appeared as particulates (300 - $400 \mathrm{~nm}$ in diameter) leaving the poly $(1,3$ DAB) surface uncovered (Fig. 3c). However, when PTFE was cast in the same situation, it enhanced the homogeneity of the film (Fig. 3d). The PTFE layer homogeneously covers the previous layer with very fine particles, and results in a flat morphology. The PTFE layer also plays a role as a good substrate for Kel-F deposition. The SEM image in Fig. 3e shows that the Kel-F layer cast onto a PTFE layer forms a more compact layer compared to the first Kel-F layer on poly(1,3DAB) (Fig. 3c). It is expected that the Kel-F layer covering the sensor over its entire surface range will reduce the oxygen effect.

Nafion has been widely used as an outer layer to reduce glucose diffusion and to improve biocompatibility in implantable miniaturized glucose sensors. ${ }^{11-14}$ When Nafion, as a biocompatible membrane, is cast on the Kel-F layer, it is

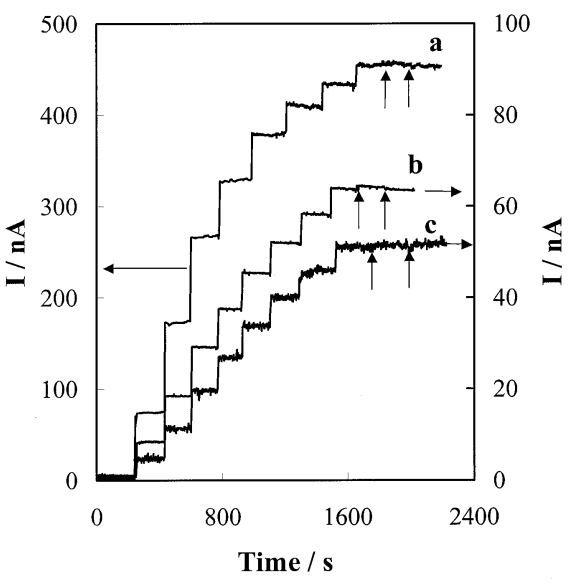

Fig. 2 The effect of outer layer as a diffusion-limiting barrier. Current responses for sensors with (a) PTFE, (b) Kel-F/PTFE/Kel-F, and (c) Kel-F/PTFE/Kel-F/Nafion in PBS. Steps correspond to the current responses of sensors on adding 1, 3, 6, 9, 12, 15, 18, and 21 $\mathrm{mM}$ of glucose. Ascorbic acid (AA) and acetaminophen (AP) were added to assess interference.

deposited more compactly with smaller particle size than Kel-F (Figs. 3e and 3f). The PTFE on the enzyme membrane are from the aqueous phase, in which PTFE particles are physically dispersed. It is no wonder that the clusters of PTFE particles deposited form the coarse PTFE membrane, if one takes account of the facts that the size of the PTFE particles is quite large and they readily aggregate to make assemblies. The Kel-F oil supposedly does not easily fill the large crevices because of its low viscosity. As a result, more analytes penetrate the PTFE membrane. It is believed that the polymer backbone of Nafion is basically hydrophobic enough to coat the PTFE as well as Kel-F. The interstitial space among the particle assemblies can be effectively stuffed by exposure to the Nafion solution. The more compact outer membrane structure is expected to slow down the rate of mass transfer, and thereby decrease the oxygen effect. Table 1 summarizes the sensitivities, currents, and response times of the two types of outer layer compositions, that is Kel-F/PTFE/Kel-F, and Kel-F/PTFE/Kel-F/Nafion. Each background current stabilizes within $30 \mathrm{~min}$ and this proved to be very reproducible, to within $1.0 \mathrm{nA}$. Sensitivities for KelF/PTFE/Kel-F, and Kel-F/PTFE/Kel-F/Nafion were $2.8 \pm 0.8$ and $1.6 \pm 0.6 \mathrm{nA} / \mathrm{mM} \mathrm{mm}^{2}$, respectively, where the response times $\left(t_{90}\right)$ of both sensors were less than $60 \mathrm{~s}$ in quiescent solution and less than $5 \mathrm{~s}$ in constantly stirred solution. Nafion has another advantage as an outermost membrane. It is soluble in a small quantity of alcohol and can be dispersed in water. Coating processes with Nafion cause minimal damage of previously immobilized enzymes, while most biocompatible hydrophobic polymers including polyurethane require organic solvents that are fatal to enzymes.

\section{Oxygen effect}

The glucose oxidase-based sensor requires oxygen as a cosubstrate to carry out the glucose oxidation. Therefore, it is important to examine the effect of oxygen upon the performance of the sensor. ${ }^{15}$ Zhang et al. investigated the relationship between sensitivity and linearity at several oxygen partial pressures, and concluded that it is essential that the sensitivity should be suppressed to such a degree that the rate of oxygen consumption by the sensor is negligible compared to the rate of tissue oxygen supply. As already mentioned, many 


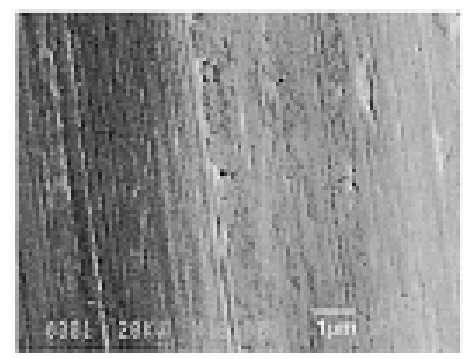

(a)

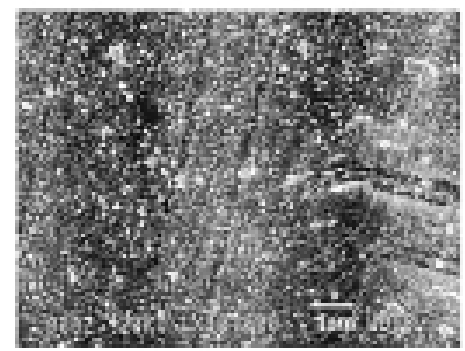

(b)

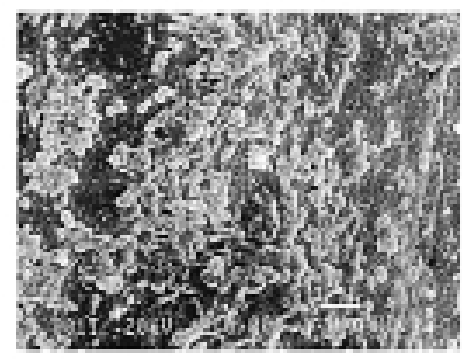

(c)

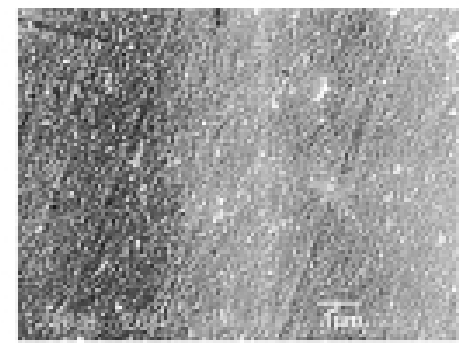

(d)

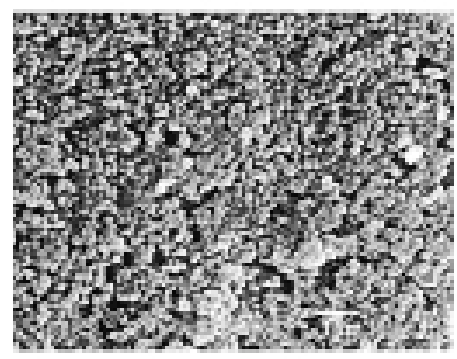

(e)

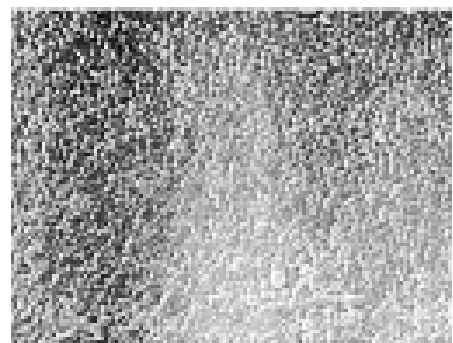

(f)

Fig. 3 SEM images showing morphological changes of the electrode surface during sensor fabrication. (a) Bare Pt; (b) after poly(1,3-DAB) was electrodeposited with immobilized $\mathrm{GO}_{x}$; (c) after coating with Kel-F; (d) after coating with PTFE; (e) after re-coating with Kel-F; (f) after a final coating with Nafion.

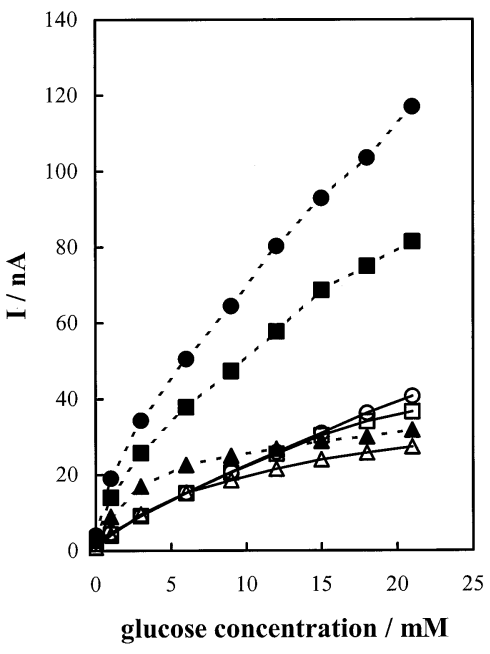

Fig. 4 Calibration curves under various oxygen tensions (air: circles, $40 \mathrm{mmHg}$ : squares, $15 \mathrm{mmHg}$ : triangles) for sensors with (filled symbols) and without (open symbols) an outer layer of $\mathrm{PTFE} / \mathrm{Kel}-\mathrm{F} / \mathrm{Nafion}$. In vitro measurements were performed in $0.1 \mathrm{M}$ PBS $\left(\mathrm{pH} 7.4,37^{\circ} \mathrm{C}\right)$ with constant stirring.

GOx-based sensors prepared by electropolymerization have suffered from problems due to a restricted linear response range.

To solve this problem, we used Kel-F oil, which has remarkable oxygen solubility. Taking advantage of this property, Wang and $\mathrm{Lu}^{3}$ constructed a first-generation enzyme electrode, which consisted of a graphite powder and Kel-F oil, operating under severely depleted oxygen conditions. Based on the suppositions that the Kel-F thin film plays an important role in the oxygen-rich membrane and also provides a glucose diffusion-limiting barrier, the Kel-F thin film was formed on the PTFE outer layer (Fig. 3e). Figure 4 shows the calibration

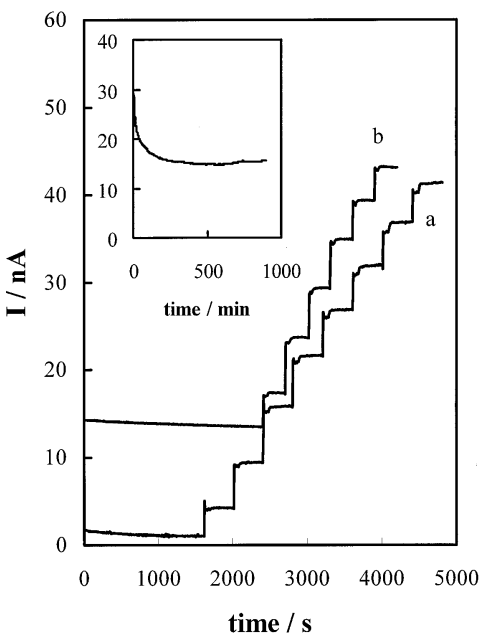

Fig. 5 Current-time responses of a glucose sensor with a Nafion outer layer (a) in PBS (at glucose concentrations of 1, 3, 6, 9, 12, 15, 18 , and $21 \mathrm{mM}$ ) and (b) in serum (at glucose concentrations of 4.6, 6 , $9,12,15,18$, and $21 \mathrm{mM}$ ). Currents during stabilization are omitted. Continuous monitoring data in serum containing $6 \mathrm{mM}$ glucose (inset).

curves obtained under various oxygen tensions for the sensors with and without an outer layer of Kel-F/PTFE/Kel-F/Nafion. The figure shows that a sensor without an outer layer is oxygendependent, whereas a sensor with an outer layer is relatively oxygen-independent. In terms of sensitivity and linearity, this result is consistent with that of Zhang et al., rather than with that of Wang et al. Zhang et al. achieved a sensor with good linearity at very low oxygen partial pressure by lowering its sensitivity by thickening the outer layer. They suggested that optimal sensitivity for sensor preparation should be in the range 
Table 1 In vitro characteristics of glucose sensors

\begin{tabular}{|c|c|c|c|c|c|}
\hline \multirow[b]{2}{*}{ Outer layer } & \multirow[b]{2}{*}{$\begin{array}{l}\text { Background current } / \text { a } \\
\text { nA mm }{ }^{-2}\end{array}$} & \multirow[b]{2}{*}{$\begin{array}{l}\text { Sensitivityb/ } \\
\mathrm{nA} \mathrm{mM} \mathrm{mm}{ }^{-2}\end{array}$} & \multirow[b]{2}{*}{$\begin{array}{l}\text { Response time } \\
\qquad\left(T_{90 \%}\right)\end{array}$} & \multicolumn{2}{|c|}{ Interference $/ \mathrm{nA} \mathrm{mm}^{-2}$} \\
\hline & & & & $\begin{array}{l}\text { Ascorbic acid } \\
(0.11 \mathrm{mM})\end{array}$ & $\begin{array}{l}\text { Acetaminophen } \\
\quad(0.17 \mathrm{mM})\end{array}$ \\
\hline Kel-F/PTFE/Kel-F & $0.8 \pm 0.4(n=7)$ & $2.8 \pm 0.8(n=7)$ & $<5 \mathrm{~s}^{\mathrm{c}}<60 \mathrm{~s}^{\mathrm{d}}$ & 0.5 & 0.0 \\
\hline Kel-F/PTFE/Kel-F/Nafion & $0.5 \pm 0.2(n=7)$ & $1.6 \pm 0.6(n=7)$ & $<5 \mathrm{~s}^{\mathrm{c}}<60 \mathrm{~s}^{\mathrm{d}}$ & 0.2 & 0.0 \\
\hline
\end{tabular}

a. Background current is stabilized within $30 \mathrm{~min}$. b. Over the concentration range $1-6 \mathrm{mM}$. c. In stirred solution. d. In quiescent solution. e. Interference is added at the maximum physiological concentration in blood.

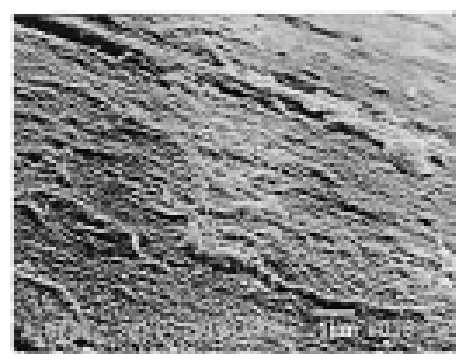

(a)

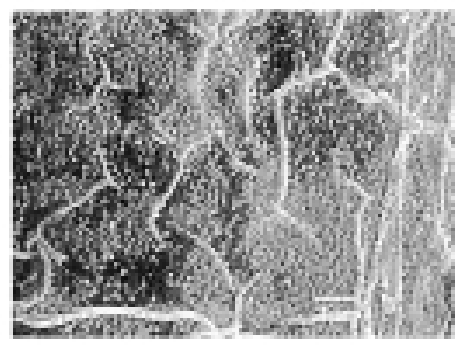

(b)

Fig. 6 SEM images of different electrode surfaces after testing in serum. (a) Pt-Ir/poly(1,3-DAB), GOx, glutaraldehyde, poly-Llysine/Kel-F/PTFE/Kel-F (Fig. 3e) after test. (b) Pt-Ir/poly(1,3DAB), GOx, glutaraldehyde, poly-L-lysine/Kel-F/PTFE/KelF/Nafion (Fig. 3f).

of $1.5-3.6 \mathrm{nA} / \mathrm{mM}$ for in vivo sensor. Considering the sensitivities of sensors with a Kel-F and a Kel-F/Nafion outer membrane in Table 1 , one can produce a practically useful glucose sensor that is free of oxygen fluctuations.

\section{Serum and in vivo tests}

In order to develop a reliable implantable glucose sensor, one must study and control the effects of the biological environment on the sensor. Therefore, the performance of the sensor with the outer layer of Kel-F/PTFE/Kel-F/Nafion was investigated in serum. In Fig. 5, current-time responses in PBS and undiluted serum are compared. The background currents were obtained from sensors stabilized for $30 \mathrm{~min}$. Longer stabilization times were required in serum than in PBS. Although the sensitivity decreased a little, the current increased in proportion to the glucose concentration as concentrated glucose solution was added, and this was stable during the serum test. After the sensors were tested in serum, their surface morphologies were examined under an SEM (Fig. 6). It was evident that decreased sensitivity was due to the adsorption of biocomponents on the sensor surface. ${ }^{13}$ The SEM images that were obtained after dipping in serum samples do not show any significant

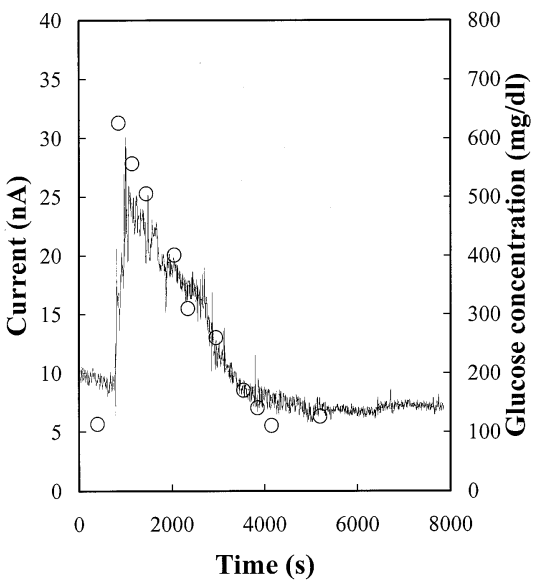

Fig. 7 Sensor signal obtained during an in vivo test (line) and glucose concentrations as determined using a glucometer (open circle).

difference between the outer membranes with and without Nafion. The observation indicates that the biocompatibility of the outer membrane is apparently not improved by coating with Nafion. After the serum test, the stability of the sensor during in vitro continuous monitoring was examined in serum containing $6 \mathrm{mM}$ glucose. The current response was stable after $15 \mathrm{~h}$ (Fig. 5 inset).

For the in vivo evaluation, a glucose sensor was implanted into the subcutaneous tissue on the back of the neck of a male Sprague Dawley rat and the output of the glucose sensor was monitored. Plasma glucose was measured independently using a Glucose Analyzer II (Beckman). In Fig. 7, the sensor current had been allowed to stabilized for $60 \mathrm{~min}$ and the figure shows the sensor response to an injection of $1 \mathrm{ml}$ of $50 \%$ glucose, and the following gradual signal decrease. It is noticeable that the lag time between changes in the blood glucose levels and the sensor response was within $1 \mathrm{~min}$ and the linearity extended to about $20 \mathrm{mM}$. In spite of the assumption of no lag time, the sensor output current correlated very well with blood glucose concentration. The sensitivity in subcutaneous tissue (in vivo sensitivity) was about $1 \mathrm{nA} / \mathrm{mM}$ and this was comparable to in vitro value. In vivo characteristics of the present glucose sensor are very promising for a one point calibration, considering that the apparent maximal rate of change of glucose in blood is 0.25 $\mathrm{mM} / \mathrm{min}^{16}$ More in vivo results will be presented in future papers.

\section{Conclusion}

We report here upon a method used to prepare a new outer layer of Kel-F film, to reduce the oxygen effect and to extend the 
linear response range of a glucose sensor prepared by electropolymerization. Output from the finished sensor was almost independent of variations in the oxygen level. The sensitivity of the fabricated sensor was $1.6 \pm 0.6 \mathrm{nA} / \mathrm{mM} \mathrm{mm}^{2}$, which is consistent with the optimal sensitivity for an in vivo glucose sensor as demonstrated by Zhang et al. That the Nafion coating enhanced biocompatibility was demonstrated by a serum test and short-term animal studies. The morphology of Kel-F/PTFE/Kel-F/Nafion was also characterized by SEM images. We believe that the Kel-F/PTFE/Kel-F/Nafion coating laminate structure shows great promise as the basis for the development of in vivo sensors based on the microfabricated electrode.

\section{Acknowledgements}

This work was supported by the Korea Science and Engineering Foundation through the Advanced Biometric Research Center.

\section{References}

1. Y. Zhang and G. S. Wilson, Anal. Chim. Acta, 1993, 281, 513.

2. D. A. Gough, J. Y. Lucisano, and P. H. S. Tse, Anal. Chem., 1985, 57, 2351.
3. J. Wang and F. Lu, J. Am. Chem. Soc., 1998, 120, 1048.

4. D. J. Strike, N. F. Derooij, and M. Koudelkahep, Biosens. Bioelectron., 1995, 10, 61.

5. M. D. Imisides, R. John, and G. G. Wallace, Chemtech, 1996, 26, 19.

6. H. Yang, T. D. Chung, Y. T. Kim, C. A. Choi, C. H. Chun, and H. C. Kim, Biosens. Bioelectron., 2002, 17, 251.

7. A. Heller, Аnnu. Rev. Biomed. Eng., 1999, 1, 153.

8. F. Palmisano, P. G. Zambonin, and D. Centonze, J. Anal. Chem., 2000, 366, 586.

9. T. D. Chung, R-A. Jeong, S. K. Kang, and H. C. Kim, Biosens. Bioelectron., 2001, 16, 1079.

10. T. Matsumoto, A. Ohashi, N. Ito, H. Fujiwara, and T. Matsumoto, Biosens. Bioelectron., 2001, 16, 271.

11. R. C. Mercado and F. Moussy, Biosens. Bioelectron., 1998 , 13,133

12. F. Moussy, S. Jakeway, D. J. Harrison, and R. V. Rajotte, Anal. Chem., 1994, 66, 3882.

13. F. Moussy, 1st Annual International IEEE-EMBS Special Topic Conference on Microtechnologies in Medicine \& Biology, 2000, October 12 - 14, Lyon, France.

14. N. Wisniewski, F. Moussy, and W. M. Reichert, J. Anal. Chem., 2000, 366, 611.

15. Z. Tang, R. F. Louie, M. Payes, K-C. J. Chang, and G. J. Kost, Diabetes Technology \& Therapeutics, 2000, 2, 349.

16. D. A. Baker and D. A. Gough, Anal. Chem., 1996, 68, 1292. 